\title{
La visión en Galicia sobre la asistencia al paciente crónico y pluripatológico; del hospital al sistema sanitario del futuro: Estrategia SERGAS 2014
}

\author{
Galician point of view on the medical care of the chronic and pluripathological \\ patient; form hospital to the sanitary sistem of the future. SERGAS 2014 strategy
}

Rocío Mosquera Álvarez', Nieves Domínguez González², Eugenia Lado Lema³, Josefina Monteagudo Romero4.

${ }^{1}$ Conselleira de Sanidad, Xunta de Galicia

${ }^{2}$ Gerente del SERGAS

${ }^{3}$ Vicegerencia del SERGAS

${ }^{4}$ Jefa de Gabinete de la Consellería de Sanidad

\section{Nuevos hospitales, nuevas realidades}

Como se ha expresado en los artículos anteriores, se precisan nuevos paradigmas en la atención a los pacientes pluripatológicos que superen la tradicional división compartimentada de la asistencia sanitaria, para lograr una asistencia integrada y eficiente, que sitúe permanentemente al paciente en el radar sanitario. Se define así la organización sanitaria integrada, cuyas características básicas se exponen en la tabla 1,2:

Tabla 1: Características básicas de la organización sanitaria integrada

a. Estratificación de la población de referencia por niveles de estado de salud.

b. Fomentar el papel de la enfermería con la asunción de nuevos roles.

c. Promoción y prevención de la salud a través de programas asistenciales gestionados.

d. Historia clínica integrada y única.

e. Prescripción electrónica única

f. Coordinación con los dispositivos sociosanitarios.

g. Proceso asistencial integrado con los centros de subagudos o de media larga estancia.

h. Asistencia no presencial con cartera de servicios (desde citación conjunta a consejo sanitario).

i. Impulso a profesionales innovadores con evaluación de resultados y traslación de proyectos.

Naturalmente ello debe conllevar una redefinición y actualización de los tradicionales roles asignados al hospital en el sistema sanitario. En este sentido, en el año 2011, la Sociedad Española de Directivos de Atención Primaria (SEDAP) publicaba en 2011 un informe titulado "Un nuevo hospital para una nueva realidad" en que diferentes Gerentes de hospitales, así como Directores Generales de Servicios de Salud (País Vasco, Castilla y León, Cantabria, Valencia, etc.. ) meditaban sobre dicha adaptación a los nuevos tiempos, enfatizando todos, como denominador común, las características de la organización sanitaria integrada expresadas más arriba. En dicha publicación, también desde Galicia, se exponían las reflexiones sobre tal tema en una colaboración titulada "Del hospital al sistema sanitario del futuro"3. Dicho artículo, debido a su autoridad, interés, actualidad y paradigma definitorio de intenciones en Galicia, se comenta aquí, con leves modificaciones, casi en su integridad.

El trabajo parte de la base de que el hospital tradicional, considerado como un santuario cerrado, estático, autogestionario y donde se ubicaban los mejores medios, la información más actualizada y los mejores expertos es una institución desfasada. Por el contrario, los hospitales actuales deben ser organismos vivos, con organizaciones complejas, pero eficientes en su actuación. Ello pasa ineludiblemente por aprovechar el potencial de sus profesionales, fomentar el trabajo en equipo e interaccionar con otras instituciones, y constituir una unidad funcional con Atención Primaria.

Tal parece la única forma de afrontar los retos actuales y futuros: poblaciones envejecidas y con pluripatología, a las que deberá prestarse atención en su domicilio o residencia, mientras que la atención hospitalaria se centrará más en pacientes críticos (trasplantes, oncológicos, grandes lesionados) y en los tratamientos ambulatorios (cirugía mínimamente invasiva, tratamientos ambulatorios).

Otro gran reto es conseguir la implicación y el compromiso de pacientes, cada vez más exigentes y con mayor acceso a la información, aunque no siempre bien informados sobre su enfermedad y las posibilidades aportadas por el sistema sanitario. La tecnología y el avance del conocimiento conducen a una Medicina más orientada a la prevención y a la promoción, en la que el ciudadano quiere una garantía en salud y bienestar más predictiva (genómica y proteómica), con tratamientos cada vez más personalizados.

Desde hace unos años las tecnologías de información y comunicación (TIC) permiten disponer y compartir -en todos los niveles de la organización y con todos los profesionales-, toda la información de los pacientes a través de la historia clínica electrónica (HCE). Ello ha permitido borrar los muros 
del hospital, al hacer accesible la información (toda la información, incluidas pruebas radiológicas, desde las más simples a las más complejas e independientemente de quien las haya solicitado, analíticas, EKG...) a todos los profesionales que puedan tener que atender a un paciente, en cualquier circunstancia y en cualquier nivel asistencial.

También permite avanzar en la organización del trabajo: trabajar por procesos, con protocolos, vías y guías clínicas comunes, con un único objetivo: hacer aquello que aporte valor al paciente. No tendrá sentido realizar más actividad, ni más consultas, ni más determinaciones analíticas, ni más exploraciones de cualquier tipo, si no le aportan algún beneficio al paciente. Para ello, la organización deberá madurar y adquirir nuevos hábitos de trabajo: no se trata de hacer más, se trata de hacer lo necesario, evitando lo innecesario (disminuyendo la variabilidad clínica) y haciendo sólo aquello que aporte valor al paciente y al sistema.

Del mismo modo que la interdisciplinariedad permite potenciar el conocimiento; las alianzas con distintos agentes e instituciones y con el sector tecnológico harán posible el óptimo aprovechamiento de los avances tecnológicos. Sería impensable un hospital de futuro que no incorpore dichos beneficios en el cuidado de la salud y que no aproveche las alianzas con los proveedores tecnológicos para hacerlo de manera más eficaz y eficiente.

Por otra parte, la situación económica actual y el previsible futuro, obligará a buscar nuevas fórmulas de colaboración y gestión que posibiliten independizar la incorporación y renovación de la tecnología y las infraestructuras del ciclo económico. Es imprescindible establecer colaboraciones y relaciones a largo plazo con los proveedores privados que permitan un compromiso real para conseguirlo. Como una apostilla en este sentido cabe decir que debido a la crisis el gasto sanitario per cápita español en 2011 cayó un 4,1\% con respecto al 2010, con reducciones sin excepción en todas las CCAA.

Pero el principal activo y valor de las organizaciones sanitarias, del hospital del presente y del futuro, son los profesionales. Es el momento del trabajo en equipo multidisciplinar, de que el profesional excelente lo sea trabajando en equipo, de que los buenos resultados sean el fruto de la integración de esfuerzos y aportaciones de todos. Los profesionales tenemos que organizarnos en torno al paciente, para prestarle la atención que necesita a lo largo de todo su proceso asistencial. En esta nueva organización del trabajo el proceso empieza y termina en Primaria, aunque en él participen profesionales de distintas especialidades. Y No debemos olvidar que el paciente es parte activa en este proceso, con quien tenemos que establecer una alianza terapéutica, fundamental para el éxito de cualquier tratamiento.
Esta alianza es y será todavía más importante de cara al futuro y a la hospitalización domiciliaria. Esto depende de un cambio en las funciones de los profesionales para dispensar cuidados, y de una solución de comunicación que dé soporte y confianza al paciente. Pero también de que el paciente asuma un nuevo papel y se responsabilice de su enfermedad y de sus cuidados, sacando el mejor partido a la disponibilidad de medios tecnológicos (acceso a un teléfono de contacto único con la enfermera o el médico responsable). El contacto personal es crítico en la relación médico/paciente, pero se pueden establecer contactos por otros canales sin perder la "humanización de los actos médicos".

Este nuevo paradigma asistencial exige cambios organizativos estructurales, en los que se redefinan los roles que jugarán los profesionales sanitarios independientemente de su procedencia. Estamos ante una revolución organizativa que por otro lado se señala como una necesidad crítica a la que tenemos que enfrentarnos. El futuro no depende de la creación de nuevos servicios que convivan con los servicios actuales, se trata de encontrar una solución transversal que pivote sobre Primaria, con un papel relevante de la enfermería, y de la que también formen parte los especialistas hospitalarios.

Este cambio organizativo no será posible sin un cambio del rol del directivo. Esta macroorganización con equipos directivos de Primaria y Hospitalaria, en la que el "equipo directivo" es el centro y a veces la razón misma de toda la organización, en la que la figura del gerente, controlador y todopoderoso, ensombrece al propio equipo, debe cambiar. La organización debe ser cada vez más horizontal, más transversal, en definitiva, más reticular. La nueva organización constará de unidades funcionales (unidades de gestión clínica), en las que se integran los profesionales, con capacidad de decisión en los temas que les afecten, incluido el gasto que generan. El papel del gerente en este nuevo sistema debe ser de facilitador y soporte, pues cada unidad debe tener autonomía, respetando siempre las reglas de juego acordadas, incluidas la valoración de resultados y responsabilidades.

Esta nueva organización no tiene garantía de futuro si no nos dotamos de un sistema retributivo más justo. Sin duda es imprescindible conseguir el pago por los resultados obtenidos, si bien es necesario garantizarles a los profesionales estabilidad profesional y un entorno de trabajo que les permita realizar una práctica clínica adecuada. También se debe considerar la feminización de la Sanidad y los nuevos roles sociales de los trabajadores sanitarios.

Todo esto refuerza la idea de que hablar de hospital es algo del pasado, el futuro debe basarse en una organización sanitaria más abierta, más flexible y más horizontal en la que se superen las barreras que separan los distintos niveles de atención, los distintos servicios clínicos basados en las espe- 
cialidades médicas, los distintos papeles entre profesionales. En definitiva, que el lugar físico deje de ser determinante en la atención al paciente, y que ésta se adapte a sus necesidades cambiantes y a la disponibilidad de medios, tanto humanos como tecnológicos o materiales.

Con esta visión en el Servicio Gallego de Salud se elaboró una hoja de ruta -la Estrategia Sergas 2014-, que contiene los principales objetivos, y líneas de actuación para alcanzarlos. Dentro de ellas, destaca la apuesta por la gestión integrada, la gestión clínica y la gestión por procesos. Para lo que se consideran herramientas imprescindibles la extensión de la historia clínica y receta electrónica a todo el sistema. Además, con el objetivo de implicar a los ciudadanos y pacientes, y facilitar su participación, se inicia la Escuela Gallega de Salud para Ciudadanos, verdadero punto de encuentro de profesionales, asociaciones y pacientes. Otras iniciativas recientes están dirigidas a la atención de pacientes crónicos y polimedicados, en las que de nuevo juegan un papel fundamental los profesionales de enfermería. En esta estrategia está siempre presente el compromiso con la gestión rigurosa y eficiente como elemento imprescindible para mantener la solvencia y sostenibilidad futura de nuestro sistema sanitario público.

En este sentido nuestras iniciativas están dirigidas a detectar ineficiencias y gastos prescindibles para garantizar las prestaciones sanitarias actuales con el nivel de seguridad y calidad adecuadas. Somos conscientes de que todos estos cambios y transformaciones sólo serán posibles contando con la implicación y complicidad de los profesionales, aunque no es menos cierto que debemos afrontar el gran reto de implantar un nuevo modelo de desarrollo profesional y una modificación profunda del sistema retributivo. En la actualidad y, en mayor medida en el futuro, el sistema sanitario no sólo será una organización prestadora de servicios sanitarios, sino también un lugar en el que se realizan actividades de docencia y de generación de conocimiento, de investigación e innovación. Por ello, todos los agentes debemos ser conscientes de que el futuro depende de nuestras decisiones de hoy. Todos somos responsables de superar los retos que nos conduzcan al sistema sanitario del futuro.

\section{La Estrategia SERGAS 2014 para la atención a pacientes ${ }^{4}$}

A partir del año 2009 y como consecuencia de la necesidad de adaptar y transformar la asistencia en el SERGAS para avanzar hacia la excelencia del sistema, se comenzó a trabajar para "definir los objetivos estratégicos y las líneas de actuación que debía acometer el SERGAS en los próximos años para lograr dicha transformación de su sistema de salud y consolidar un modelo sanitario innovador, centrado en los ciudadanos, y que asegure la continuidad asistencial, la seguridad, la eficiencia y la implicación de los profesionales". Se definieron una serie de fases en este proceso: 1. Organización del trabajo para recabar toda la información y documentación necesaria, y articular la forma de participación en la elaboración del plan estratégico. 2. Revisión de la estrategia global del Sergas, definición del punto de partida, del contexto nacional e internacional y tendencias actuales del sector sanitario, 3. Elaboración del mapa de procesos del Sergas, con un análisis de las oportunidades de mejora, definición de líneas de actuación y estudio de las mejoras prácticas de otras CCAA 4. Diagnóstico de situación de los macroprocesos con análisis en profundidad de éstos para identificar las oportunidades de mejora y las líneas futuras de actuación 5. Definición de líneas de actuación explicitando misión, visión, valores y objetivos estratégicos.

En todo este proceso participaron más de 140 profesionales y expertos de la Consellería de Sanidad, del SERGAS, de los hospitales, de atención primaria, de Sociedades científicas, Colegios profesionales, y Organizaciones sindicales y políticas.

El resultado final fue la Estrategia SERGAS 2014: La sanidad pública al Servicio del paciente en la que se definen los 7 objetivos estratégicos priorizados y las 40 líneas de actuación que deben desarrollarse para alcanzarlos.

Su conocimiento y difusión es importante, porque dicho plan constituye la hoja de ruta para llevar a la práctica los principios enumerados anteriormente, y conseguir una asistencia lo más racional y eficiente posible. A continuación se detallan casi literal y detalladamente los objetivos y acciones estratégicas específicamente relacionados con la asistencia a los pacientes con enfermedades crónicas y grupos especiales de población como los ancianos (Objetivo 1 con sus acciones estratégicas). Para situar en la perspectiva adecuada tales estrategias, que guardan indisoluble relación con el diseño global del Plan, se hace mención sumaria al resto de objetivos y líneas estratégicas contenidos en dicho plan.

\section{ESTRATEGIA SERGAS 2014.}

\section{La Sanidad pública al servicio del paciente}

OBJETIV0 1. Promover la salud y responder a las necesidades del ciudadano a través de dispositivos asistenciales y planes específicos.

\section{Líneas estratégicas.}

1.1. Estructura de gestión integrada con el objetivo general de lograr una atención integral de los problemas de salud de los ciudadanos, eliminando las barreras entre los diferentes niveles asistenciales y superando la fragmentación de la asistencia.

Elementos clave e implicaciones: 
- Desaparición de barreras entre niveles asistenciales, considerando que la asistencia es un proceso horizontal, sin rupturas. Requiere la actuación interdisciplinar y coordinada de los diferentes ámbitos para garantizar continuidad asistencial, calidad y eficiencia.

- Los profesionales de la estructura organizativa de gestión integrada serán responsables de la gestión unitaria de todos los recursos de su ámbito y de las prestaciones y programas que se desarrollen, quedando adscrita a ella la totalidad de dispositivos y recursos sanitarios y sociosanitarios, sin alterar el mapa sanitario vigente.

La estructura de gestión integrada tendrá como objetivos:

1. Centrar la atención en el paciente con implicación de todos los profesionales en la toma de decisiones, mediante la gestión clínica y la gestión por procesos

2. Garantizar la equidad, accesibilidad, continuidad, calidad y seguridad de la atención, mediante la gestión por procesos, con priorización según criterios clínicos.

3. Minimizar la variabilidad clínica y eliminar la duplicidad de procedimientos diagnósticos y terapéuticos mediante la implantación de las recomendaciones contenidas en las guías y vías de práctica clínica.

4. Garantizar la transparencia en la información y la autonomía de decisión de los pacientes.

5. Orientar la planificación de los procesos en torno a los problemas de salud.

6. Utilizar eficientemente todos los recursos mediante la promoción del trabajo en equipo y la implicación de profesionales en la gestión.

Se desarrollarán actuaciones encaminadas a:

- Definir la nueva estructura organizativa de gestión integrada de los servicios periféricos del SERGAS.

- Integración funcional de estrategias, procesos asistenciales integrados, sistemas de información y gestión de recursos.

- Desarrollar los procesos asistenciales integrados.

- Lograr la implicación de los profesionales en los objetivos definidos.

- Incrementar la capacidad resolutiva:

*Redefinición de la cartera de servicios, potenciando específicamente la telemedicina y atención domiciliaria.

*Incorporación de medios diagnósticos que aumenten la capacidad de resolución en los centros de salud, consultas de hospital, urgencias hospitalarias y puntos de atención continuada.

- Orientar los Acuerdos de Gestión (ADX) hacia la gestión clínica e identificar objetivos globales implicando a los profesionales en su consecución.
- Implantar un sistema de financiación de acuerdo al modelo de gestión integrada.

- Incorporar la atención sociosanitaria en el modelo de gestión integrada.

- Establecer un sistema de información adaptado al nuevo modelo de gestión.

\section{2. Modelo de gestión de enfermedades crónicas.}

Con el objetivo general de hacer que el paciente continúe en la pantalla del radar del sistema sanitario, reorientando éste hacia la gestión integrada de enfermedades crónicas, bajo un enfoque de gestión por procesos que redunde en mejores resultados clínicos, adherencia a los tratamientos, responsabilidad del paciente con su enfermedad y mejora de la calidad de vida.

Elementos clave e implicaciones:

Supone un enfoque específico para cada grupo de pacientes con enfermedad crónica, prestando especial atención a pacientes pluripatológicos y a pacientes con cáncer.

Los programas de intervención se desarrollarán atendiendo a una estratificación de pacientes según su nivel de complejidad:

1. Pacientes enfermos crónicos con buen autocontrol de su enfermedad, con diseño de intervenciones enfocadas a apoyarles en la autogestión de su enfermedad.

2. Pacientes con patologías con morbilidad intermedia y alto uso de recursos, con diseño de intervenciones enfocadas a la gestión de patologías.

3. Pacientes muy complejos, con alta comorbilidad y alto uso de recursos, con diseño de intervenciones enfocadas a la gestión de casos.

El desarrollo de un modelo de gestión de enfermedades crónicas implica:

- Evaluar necesidades físicas, sociales y psicológicas, para diseñar un paquete integral de cuidados.

- Definir las actuaciones de los diferentes niveles.

- Definir procesos asistenciales específicos, utilizando protocolos, guías y vías clínicas, con sistemas proactivos de seguimiento, para lograr adherencia al tratamiento y evitar agudización de la enfermedad.

- Adaptar los sistemas de información a la gestión de enfermedades crónicas.

- Redefinir los roles de los profesionales, con mayor protagonismo a la enfermería (en especial la de enlace), particularmente en la gestión de casos y en la atención a domicilio.

- Utilizar dispositivos innovadores que permitan la gestión de enfermedades crónicas, en la atención domiciliaria y de conexión de pacientes con profesionales sanitarios 
(atención no presencial, envío de resultados clínicos, etc.)

- Identificación de mejores prácticas y realización de análisis comparativos.

- Superar un modelo diseñado para pacientes agudos, y transformarlo en un sistema capaz de respuesta a los pacientes crónicos, donde sean identificados, tratados 0 dados de alta con un plan preventivo y de tratamiento, y que sean biomonitorizados.

\subsection{Atención a colectivos específicos con necesidades}

diferenciadas: ancianos (tabla 2), mujer, niños y salud mental con el objetivo general de prestar una atención integral dirigida a dichos colectivos, que necesitan una atención diferenciada, introduciendo en el sistema la sensibilidad ante las diferentes necesidades.

Tabla 2: Atención al paciente anciano:

Crear pautas de funcionamiento de equipos multidisciplinares que proporcionen una visión bio-psico-social del paciente anciano

Trabajar de forma coordinada con la Consellería de Trabajo y Bienestar, de cara a incorporar la atención social en un modelo de atención integral al paciente anciano, estableciendo dispositivos de seguimiento ambulatorio

Desarrollar un programa específico orientado a mejorar la salud y la calidad de vida de los pacientes mayores polimedicados.

Incorporar técnicas de gestión de casos, potenciando la figura de la enfermera.

Impulsar la atención domiciliaria en la atención al paciente anciano.

Dentro de este primer objetivo también son importantes las siguientes líneas estratégicas:

1.4. Integración de la atención sociosanitaria con el objetivo general de atender de forma integrada las necesidades de la población en el ámbito sociosanitario, bajo criterios de igualdad, equidad, accesibilidad, universalidad y complementariedad de las acciones de las diferentes administraciones públicas.

Para ello se enumeran una serie de acciones como la elaboración de un mapa de recursos sociosanitarios, y su coordinación para una asistencia más eficiente, se incorporarán las necesidades sociales a la historia electrónica y se estratificará el grado de dependencia, se potenciará la permanencia en el entorno familiar, evitando la institucionalización siempre que sea posible.

1.5. Garantía de accesibilidad con el objetivo general de mejorar la accesibilidad de los ciudadanos al sistema sanitario gallego; aprovechando las posibilidades que ofrecen las nuevas tecnologías de la información y de la comunicación.

Potenciar servicios a través de diversos canales (teléfono, web, SMS), para facilitar la tramitación electrónica de diversos servicios como citas y consultas. En definitiva, se trata de potenciar la atención integrada mediante herramientas informáticas adecuadas como la historia y receta electrónicas.

\subsection{Impulso a los programas de prevención y promoción} de la salud con el objetivo general de desarrollar actuaciones encaminadas a la promoción de estilos de vida saludables y al desarrollo de políticas de prevención de la enfermedad.

Para ello se impulsan programas de prevención y promoción de salud desde los centros sanitarios, reforzando el papel de la enfermería, y dando especial relevancia a la prevención de enfermedades crónicas, consumo de alcohol, tabaco y otras drogas, cánceres de mama, cérvix o colon, riesgos hospitalarios, etc. Además, se imparten, cursos de formación a través de la Escuela Gallega de Salud para Ciudadanos para fomentar los autocuidados, la mayor responsabilidad del paciente y la promoción de hábitos de vida saludables (ejercicio físico, hábitos dietéticos....).

1.7. Participación de los ciudadanos y de los pacientes mediante la Escuela Gallega de Salud para Ciudadanos, las encuestas de satisfacción, el desarrollo de estructuras de participación comunitaria formales como el Consejo Gallego de Salud, los Consejos de Salud de Área y las Comisiones de participación ciudadana, cauces de participación para sugerencias y opinión ciudadana.

\subsection{Desarrollo eficaz del derecho de libre elección de médico y hospital.}

Los otros objetivos y acciones estratégicas contemplados en la Estrategia Sergas 2014 con incidencia también en la atención a pacientes pluripatológicos, son:

OBJETIVO 2. Garantizar la prestación de servicios en el lugar y momento adecuados, con niveles de calidad y seguridad.

Líneas estratégicas: 1. Procesos asistenciales integrados. 2. Garantía de atención en tiempo y forma según patologías definidas. 3. Procesos de alta resolución. 4. Atención domiciliaria y hospitalización a domicilio 5. Cuidados paliativos, 6. Política de calidad y seguridad del paciente. 7. Mejores prácticas de prescripción y dispensación de medicamentos. 8. Colaboración con los centros privados.

OBJETIV0 3. Definir una política de personal alineada con las necesidades del sistema y de los profesionales.

Líneas estratégicas: 1. Nuevo marco de gestión de recursos humanos. 2. Desarrollo profesional, 3. Excelencia docente. 4. Gestión del conocimiento. 5. Gestión clínica. 6. Comunicación con los profesionales

OBJETIV0 4. Desarrollar unos sistemas de información que faciliten la práctica clínica y la toma de decisiones.

Líneas estratégicas: 1. Implantar la historia clínica y receta electrónica en todos los centros sanitarios. 2. Proporcionar a todos los profesionales herramientas que permitan aumentar la eficiencia, la calidad y la capacitación en el trabajo. 3. 
Adaptar los sistemas de información hacia la gestión integral de los problemas de salud. 4. Garantizar la disponibilidad, seguridad, confidencialidad y calidad de la información sanitaria OBJETIV0 5. Hacer que el sector sanitario actúe como motor de crecimiento económico e impulsar alianzas con todos los agentes implicados.

Líneas estratégicas: 1. Promoción de la investigación, innovación y transferencia de resultados. 2. Responsabilidad Social Corporativa. 3. Desarrollo del clúster de salud de Galicia. 4. Impulso a las relaciones internacionales. 5. Marco de relaciones con instituciones y grupos sociales

OBJETIV0 6. Gestionar los recursos de forma rigurosa y eficiente.

Líneas estratégicas: 1. Establecimiento de un marco de rigor y austeridad en el sistema sanitario público de Galicia. 2. 3. Plataformas de diagnóstico por imagen y laboratorios clínicos. 4. Central de compras y plataforma logística 5. Nueva estrategia corporativa de transporte sanitario. 6. Cartera de servicios, técnicas, procedimientos y referencias del Servicio Gallego de Salud.

OBJETIV0 7. Modernización de las infraestructuras sanitarias.

Líneas estratégicas: 1. Nuevas fórmulas de financiación y gestión de infraestructuras sanitarias. 2. Construcción y puesta en funcionamiento de los nuevos hospitales y centros de alta resolución. 3. Construcción y reforma de centros de salud. 4. Renovación tecnológica

\section{Bibliografía}

1. García Prieto EE. El hospital del siglo XXI. En: Informe de la Sociedad Española de Directivos de Atención Primaria (SEFDAP). Un nuevo hospital para una nueva realidad. Madrid: SANED, 2011, pp. 101-108. Disponible en www.sedap.es, consultado 28/01/2012)

2. Pérez Gil J. Hospital del futuro. Ibídem, pp. 132-40.

3. Mosquera Álvarez R, Eugenia Lado Lema E, Josefina Monteagudo Romero J. Del hospital al sistema sanitario del futuro. En: Ibídem, pp. 122-125, consultado, 28/01/2012).

4. Estrategia SERGAS 2014. La Sanidad Pública al servicio del paciente. Disponible en: http://www.sergas.es/Docs/Conselleria/Estrategia_Sergas_2014.pdf, consultado 30/01/2011. 